\title{
Flux-Locked Current Source Reference
}

\author{
Weston L. Tew, Member, IEEE, and Edwin R. Williams, Member, IEEE
}

\begin{abstract}
The quantization of flux in a closed superconducting circuit is used to provide a stable reference current. A 10 $\mathbf{m A}$ current source is coupled through a toroidal transformer to a dc SQUID input, and the resulting signal is fed back as an error current. The result is a net flux linkage that exhibits shortterm stability of 1 part in $10^{9} / \mathrm{h}$. The net current is quantized with a step size of $59.4 \mathrm{nA}$, and it will exhibit the same stability as the flux provided the mutual inductance of the transformer remains constant. This current is passed through a precise 100 $\Omega$ resistor and compared against Zener diode references. The observed temperature coefficient for the flux transformer is 28.5 $\pm 3 \mathrm{ppm} / \mathrm{K}$ at $4.2 \mathrm{~K}$. Possible sources for the temperature dependence are discussed.
\end{abstract}

\section{INTRODUCTION}

$\mathrm{T}$ HE difficulty in creating a highly stable and predictable dc secondary voltage reference is a long-standing problem in electrical metrology. Such references are typically needed as calibration transfer standards between the Josephson array primary standard and other secondary voltage references. Most Zener diode-based voltage references exhibit short-term noise that limits calibration accuracy to a few parts in $10^{8}$ [1]. Short-term noise is lower in transfer resistance standards than in Zener references, and their values are generally predictable to a few parts in $10^{8}$ over long periods [2]. Hence, a high-stability current source in series with such a resistor can serve as a voltage reference with stability comparable to that of the current.

The ideal suitability of a superconducting quantum interference device (SQUID) system for current controllers has long been recognized [3]. The technique of locking an external dc current to the magnetic flux from a persistent superconducting current was first demonstrated by Gallop [4]. Weyand has constructed similar systems using second-order magnetic gradiometers as flux transformers [5]. As an alternative technique, smaller currents may be directly injected into a SQUID input and comparably stabilized. This approach was realized at the Electrotechnical Laboratory by Sakamoto et al. [6].

Manuscript received June 12, 1992; revised September 21, 1992. This work was supported in part by the National Research Council Postdoctoral Associateship Program at NIST.

W. L. Tew was with the Electricity Division, National Institute of Standards and Technology, Gaithersburg, MD 20899. He is now with the Process Measurements Division, National Institute of Standards and Technology, Gaithersburg, MD 20899.

E. R. Williams is with the Electronics and Electrical Engineering Laboratory, Electricity Division, National Institute of Standards and Technology, U.S. Department of Commerce, Technology Administration, Gaithersburg, MD 20899.

IEEE Log Number 9206506.
With or without an intermediate transformer or persis tent current, the external current is essentially being locked to a particular level of flux quanta and will exhibit stepwise stability. For this reason, we refer to these systems as flux-locked current sources (FLCS). Short-term stability of $10^{-9} / \mathrm{h}$ is possible in net flux linkage in most cases when the systems are built around suitable commercial SQUID detectors. The system described here in corporates some of the features of each of the above examples. In addition, we endeavor to evaluate some of the principal limitations to this approach and, in particular, the size of the temperature coefficient.

\section{Design Considerations}

Consider a closed superconducting circuit such as would be used for a dc flux transformer. The quantity that is quantized in this circuit is the fluxoid $\Phi_{S}$ defined as

$$
\Phi_{S}=\iint B \cdot d A+\mu_{0} \lambda_{L}^{2} \int J_{S} \cdot d l
$$

where $B \cdot d A$ is the magnetic flux linking the circuit, $J_{S}$ is the circulating supercurrent, and $\lambda_{L}$ is the London penetration depth. The first term is by far the dominant contribution in any macroscopic circuit, and contains the magnetic signal to which an external current would be coupled. The second term represents the contribution from the screening currents for which $J_{S} \propto\left(\lambda_{L}\right)^{-1}$. Since the sum of the two terms is quantized, changes in the distribution of screening currents can appear as changes in the flux. Thus, the flux linkage can be temperature dependent for finite values of $J_{S}$ through the temperature dependence of $\lambda_{L}$. It is then advantageous to operate a superconducting reference circuit at $J_{S}=0$.

To couple the flux of the external circuit to the superconducting circuit, a toroidal geometry offers several advantages. The complete enclosure of flux in an ideal toroidal current sheet is desirable for rejection of common mode fields and relative immunity to dimensional changes in the external circuit windings. In practice, a wire-wound toroid only approximates this ideal current distribution since some flux leakage occurs between the individual windings. The total self-inductance $L_{t}$ of a real wirewound toroid of rectangular cross section can be written as the ideal current sheet inductance $L_{S}$, less a term representing the flux linking each separate turn of wire $L_{N}$, and a small term $L_{w}$ for the contribution from flux within the wire itself. These terms are

$$
L_{t}=L_{S}-L_{N}-L_{w}
$$




$$
\begin{aligned}
L_{S} & =\frac{\mu_{0}}{2 \pi} N^{2} h \ln \frac{b}{a} \\
L_{N} & =0.332 \frac{\mu_{0}}{2 \pi} N l
\end{aligned}
$$

and

$$
L_{w} \approx \frac{\mu_{0}}{2 \pi r} N l \lambda
$$

where $N$ is the number of turns, $l$ is the wire length per turn, $r$ is the wire radius, $h$ is the toroid height, and $a$ and $b$ are the inner and outer toroidal radii [7]. For most practical dimensions, $L_{N}$ can be a few percent of $L_{t}$. Thus, as a supplement to the transformer, it is desirable to use overlapping, insulated superconducting shielding, as is standard practice in cryogenic current comparators [8]. This significantly improves the common mode rejection of the transformer to all but purely azimuthal field components [9]. The term in $L_{w}$ as given in (2d) is sometimes called the "kinetic inductance,"' although for bulk linear superconductors, it is actually half magnetic and half kinetic [10].

This configuration is essentially that of a "single-arm" cryogenic current comparator (CCC). Unlike the CCC which operates close to a flux null, the FLCS must operate off null at some finite flux linkage. Any temperature dependence through $\lambda(T)$ will be proportional to this net flux linking the reference coil.

An important parameter of a FLCS is the current step size $I_{0}$. This is simply a function of the flux quantum $\Phi_{0}$, the SQUID input coupling efficiency or mutual inductance $M_{S C}$, and the current gain $G_{i}$ of the flux transformer or

$$
I_{o}=\frac{\Phi_{0}}{G_{i} M_{S C}} .
$$

Typically, $M_{S C} \approx 20 \mathrm{nH}$ or about $10 \Phi_{0} / \mu \mathrm{A}$. This step size should be as small as is practical given the size of the reference current to be stabilized and our ability to resolve the steps with a precise resistor and digital voltmeter (DVM). It is perhaps most important that the current source in the open-loop state have short-term fluctuations that are small compared to the chosen step size in order that the flux-locked loop remains locked. Thus, a few parts per million is a practical lower limit.

The standard equivalent circuit representation of a flux transformer coupling into a SQUID input is given in Fig 1. The current gain of the transformer is given by

$$
G_{i}=\frac{M_{12}}{L_{c}+L_{2}}
$$

where $M_{12}$ is the mutual inductance between the primary and secondary windings, and $L_{c}+L_{2}$ is the total series inductance of the secondary circuit, the primary winding $L_{1}$ plus the SQUID coupling coil $L_{c}$. In the current sheet approximation of pure toroidal coupling, we have

$$
M_{12 s}=\frac{\mu_{0}}{2 \pi} N_{1} N_{2} l_{2} \ln \left(b_{2} / a_{2}\right)
$$

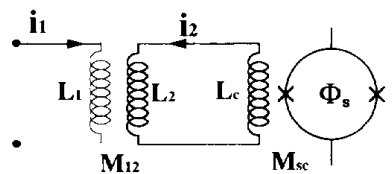

Fig. 1. Equivalent circuit for the flux transformer. $M_{12}$ is a toroidal mutual inductance.

which is the well-known property of toroidal transformers being dependent only on the dimensions of the inner or secondary winding. Hence, only the secondary winding need be designed with exceptional stability in mind.

Given a current in the primary of $i_{1}=i_{10}+\delta i_{1}$ and in the secondary of $i_{2}=i_{20}+\delta i_{2}$, then for $i_{20}=0$, we have

$$
\delta i_{2}=G_{i}\left(\delta i_{1}+i_{10} \frac{\delta M_{12}}{M_{12}}\right)
$$

where the first term is the control signal and the second term is the transformer error. The mutual inductance $M_{12}$ then becomes the artifact reference that must remain constant in order for the current to remain constant.

\section{EXPERIMENTAL SYSTEM}

The flux transformer is formed by a pair of toroidal windings. The external current passes through the primary winding, and the secondary winding is connected to the SQUID input. The secondary winding is a single layer of 120-turn NbTi alloy wire of $125 \mu \mathrm{m}$ diameter wound under tension onto a notched machineable glass-ceramic (MGC) former. A return loop to eliminate the net turn in the phase of the toroid winding is placed near the center of the former. The former has a square cross section with an inner diameter of 40 and an outer diameter of $36 \mathrm{~mm}$. This winding is surrounded by an overlapping, insulated $\mathrm{Pb}$ foil shield. The primary winding is 600 turns of polytetrafluoroethylene-insulated $\mathrm{Cu}$ wire in four alternating layers of 150 turns each, which at $4 \mathrm{~K}$ has a resistance of less than $1 \Omega$ and a self-inductance of about 70 $\mu \mathrm{H}$. The complete transformer has a dc current gain of $G_{i}$ $\approx 1.85$ at $4.2 \mathrm{~K}$. The total flux linkage at $i_{10}=10 \mathrm{~mA}$ and $M_{12}=6 \mu \mathrm{H}$ is approximately $30 M \phi_{0}$, corresponding to a mean flux density of about $55 \mu \mathrm{T}$.

A small section of the input circuit is thermally coupled to a heater element by winding three twisted pair turns around one end of a $1.3 \mathrm{~cm}$ diameter sapphire bobbin. The heater is formed from several turns of twisted pair $\mathrm{Ni}-\mathrm{Cr}$ wire with approximately $100 \Omega$ resistance at the other end of the bobbin. The entire bobbin is nested inside a MGC cylinder to insulate it from the helium bath. This allows the temperature of the section of superconductor to be elevated above $T c$ with less than $1 \mathrm{~W}$ applied power in several seconds. This thermal switch ensures that any persistent current will decay under a resistive condition in the circuit so that $i_{20} \approx 0$.

A block diagram of the system is shown in Fig. 2. The main current source (CS1) is a high-gain $10 \mathrm{~mA}$ unipolar source with a $5.4 \mathrm{~V} \mathrm{Hg}$ battery reference. It has a drift 


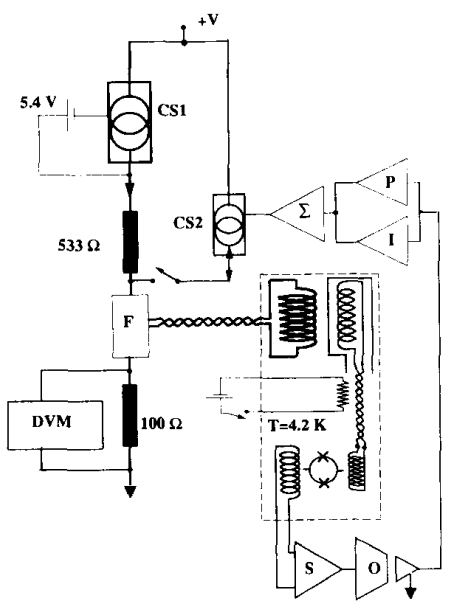

Fig. 2. System block diagram (see text).

rate of 1-5 ppm $/ \mathrm{h}$ and a noise density of about 2 $\mathrm{nA} / \sqrt{ } \mathrm{Hz}$. A single-pole passive filter $(F)$ with a $3 \mathrm{~dB}$ point of $30 \mathrm{~Hz}$ is required at the primary input in order to maintain a suitable signal-to-noise ratio. The error signal is derived from the SQUID $(S)$ output with a typical sensitivity of $8.7 \mathrm{~V} / \mu \mathrm{A}$. The signal is then fed back through an optically coupled isolation amplifier $(O)$. A parallel proportional $(P)$ and integral $(I)$ stage is summed $(\Sigma)$ into the input of a bipolar current source (CS2) which feeds the signal back as a current returning to ground. In closedloop operation, the drift of the $\mathrm{Hg}$ battery is compensated by the charging of the integrator. With proper frequency compensation from the $P / I$ stage, it is possible to achieve loop gains of $30 \mathrm{~dB}$ at $f \leq 1 \mathrm{~Hz}$ and maintain stability.

\section{RESULTS}

Our system has a step size of $59.4 \mathrm{nA}$ or about $6 \mathrm{ppm}$. The primary current source CS1 is adjustable over a range of $547 \mathrm{ppm}$ or $92 I_{o}$. The step can be determined only by accurately measuring the potential drop across the precision resistor. Thermal offsets can be cancelled by reversing the current through the resistor. The current may be set in an open-loop condition so as to lock on to any one of the accessible steps when the loop is closed and both integrators are reset. Fig. 3 is a plot illustrating a few of the current steps over a small range of the CS1 adjustment. Once locked, the closed-loop error signal indicates that the net current drift is less than $10 \mathrm{pA}$ in several hours. Fig. 4 is a plot of the error signal from the SQUID output over several hours of continuous locked operation. Occasional RF interference can momentarily unlock the system and allow the current to jump by multiples of $I_{o}$. However, it is always possible to reset the integrators and relock onto the same step. Typically, the system can remain continuously locked for more than $24 \mathrm{~h}$ with allbattery power supplies.

The most accurate measurements have been made by comparison of the voltage drop across the resistor with a

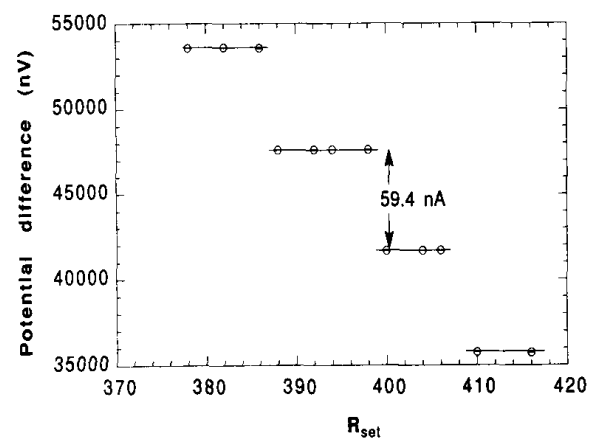

Fig. 3. A few current steps of approximately $6 \mathrm{ppm}$ spacing versus the open-loop current adjustment scale $R_{\text {set }}$. The range of $R_{\text {set }}=0-1000$ corresponds to $547 \mathrm{ppm}$ or 92 steps. The potential difference is between the $i R$ drop and the Zener reference.

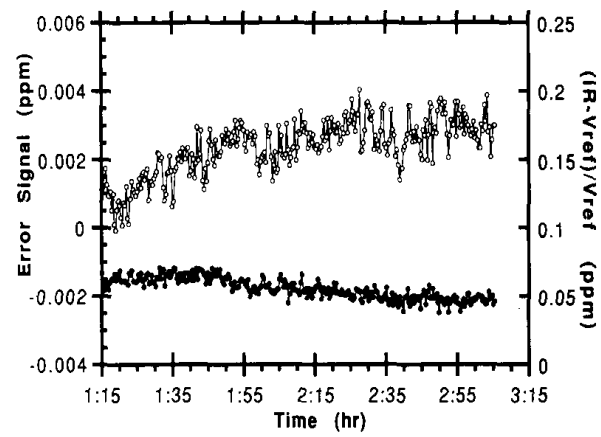

Fig. 4. Error signal as detected by the SQUID and difference voltage between the $i R$ drop and the Zener reference as read on the DVM. The top trace (right scale) is the DVM, and the bottom trace (left scale) is the SQUID. The ranges of two scales differ by a factor of 25 .

commercial Zener diode reference using a digital nanovoltmeter. The second trace in Fig. 4 shows such a difference. Under most circumstances, the short-term variations (time scale $<2 \mathrm{hr}$ ) are the same size that we measure when comparing two Zener references against each other $(\leqslant 50 \mathrm{nV})$, indicating that the Zener reference is a limitation of the evaluation. However, the value of the current can drift by much larger amounts according to changes in the liquid helium bath temperature.

A typical observed temperature dependence of the current is shown in Fig. 5. The temperature is measured by a Ge thermometer mounted near the transformer, but outside a superconducting shield. The average slope is about $28.5 \mathrm{ppm} / \mathrm{K}$. This value is reproducible from one cooldown to the next to within $\pm 3 \mathrm{ppm} / \mathrm{K}$. Slightly larger values are measured when the current is left on during a cool-down. In terms of flux variations as detected by the SQUID via the particular input inductance used here, the ratio $M_{S C} /\left(L_{c}+L_{2}\right) \sim 0.006$ attenuates the variations in the transformer to give approximately $5 \phi_{0} / \mathrm{K}$. This value has been directly verified under open-loop conditions. It has also been verified that when all flux linkage is removed, by turning off the external current and activating 


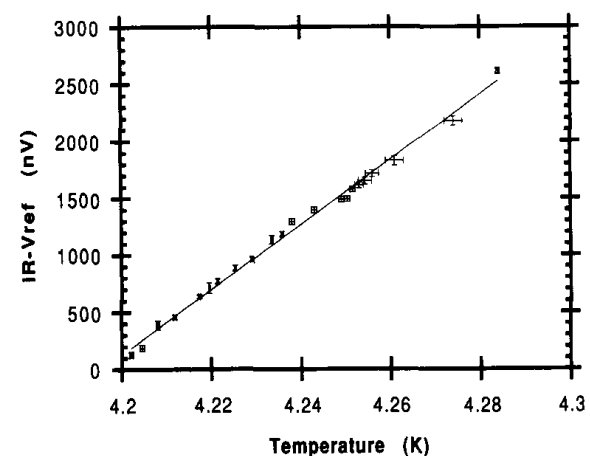

Fig. 5. Temperature dependence of the current as read by the DVM as a function of the liquid helium bath temperature. The slope of the linear fit is $28.5 \mathrm{ppm} / \mathrm{K}$.

the thermal switch, the SQUID output variations with temperature are decreased by more than a factor of 40 . We thus conclude that the flux transformer itself has a temperature coefficient of $28.5 \pm 3 \mathrm{ppm} / \mathrm{K}$.

\section{DISCUSSION}

Since changes in the dimensions of the secondary winding will produce a transformer error, we need to consider the expansion coefficient of the transformer. MGC is a typical choice for coil formers as it is known to have a linear expansion coefficient of only $1.5 \times 10^{-9} \mathrm{~K}^{-1}$ at $4 \mathrm{~K}$ [11]. The total integrated thermal contraction from $300 \mathrm{~K}$ to $4 \mathrm{~K}$ is $-1.7 \times 10^{-3}$, and a typical value for $\mathrm{Nb}-\mathrm{Ti}$ alloys is $-1.9 \times 10^{-3}$. Thus these two materials are well matched, and a tightly wound single-layer winding of NbTi on MGC should take on the dimensional stability of the MGC. We conclude that thermal expansion will not be significant contribution to the transformer error.

The superconducting penetration depth $\lambda(T)$ is temperature dependent. Also, while small, the effect of $\lambda(T)$ on the inductance of superconducting circuits is measurable [10]. It has been previously suggested that this effect should be the dominant limitation to the stability of an FLCS [4]. However, quantitative agreement between experiment and theory is generally lacking [12]. There are several experimental uncertainties that enter, most notably those concerning the condition of the surface of the superconductor. Generally, both surface roughness and oxygen enrichment in surface layers of $\mathrm{Nb}$ can increase the value of both $\lambda(T)$ and its derivative [13]. Oxygen enrichment has been shown to be associated with cold working the material.

Despite these difficulties, it is still possible to make a rough estimate of the value of a temperature coefficient of inductance $\alpha_{M}$ from $\lambda(T)$ where we adopt the definition

$$
\alpha_{M} \equiv \frac{-1}{M_{12}} \frac{\partial M_{12}}{\partial T} \approx \frac{M_{12 w}}{M_{12 s}} \frac{1}{\lambda} \frac{\partial \lambda}{\partial T}
$$

where $M_{12 w}$ is the contribution from the flux in the wire
The approximate form for $\lambda(T)$ is the Gorter-Casimir (GC) function

$$
\lambda(T)=\frac{\lambda_{0}}{\left(1-t^{4}\right)^{1 / 2}}
$$

for reduced temperature $t=T / T_{c}$ that usually remains accurate for $t>0.5$. Below this temperature, corrections are required that take into account the temperature dependence of the energy gap [12]. This has the effect of slightly increasing $\partial \lambda / \partial T$. Taking only the leading term in the derivative of the GC function, we have

$$
\frac{1}{\lambda_{0}} \cdot \frac{\partial \lambda}{\partial T} \approx \frac{2}{T_{c}} t^{3}
$$

Values of $\lambda_{0}$ for "dirty" alloys of NbTi of Ti concentration $>40 \%$ are typically about $200 \mathrm{~nm}$. From the measured residual resistivity of the particular NbTi alloy used here $(\rho \approx 80 \mu \Omega \cdot \mathrm{cm})$, we calculate from the GinzbergLandau Theory [14] in the dirty limit that $\lambda_{0} \approx 184 \mathrm{~nm}$.

It is difficult to make accurate estimates of the flux density close to the surface of a superconducting wire without doing the full boundary value problem, which is beyond the scope of this paper. For our purposes here, it is sufficient to make some simple approximations in lieu of a detailed calculation of the magnetic coupling in order to estimate $M_{12 w} / M_{12 s}$. If we neglect the effect of internal screening currents in the wire and consider only the flux contribution from the transport current, we estimate $M_{12 w} / M_{12 s} \sim 8 \lambda a / h^{2}$. Accordingly, a rough estimate from (7) and (9) for the temperature coefficient would be

$$
\alpha_{M} \approx \frac{8 \lambda a}{h^{2}} \frac{2}{T_{c}} t^{3}
$$

With values given above for the various parameters, taking $T_{c}=9.7 \mathrm{~K}$ and $t=0.433$, we calculate $\alpha_{M} \approx 30$ $\mathrm{ppm} / \mathrm{K}$. However, given the various approximations used, this agreement between the calculation and the observed temperature coefficient should be viewed as somewhat fortuitous.

\section{Conclusions}

While not a fundamental limitation to the FLCS, the observed temperature coefficient is inconveniently large. The required temperature stability of $\delta T \leq 40 \mu \mathrm{K}$ for $\delta i$ $\leq 10^{-9}$ is achievable in an $\mathrm{He}^{4}$-based system, but the present system does not incorporate the necessary features to realize this level of precision. While choosing to operate at $2 \mathrm{~K}$ rather than $4.2 \mathrm{~K}$ would have obvious advantages from the point of view of temperature stability, the loss of the convenience of a freely convecting system vented to atmosphere would be undesirable. The equilibrium bath temperature of such a vented Dewar can be expected to drift over a range of about $50 \mathrm{mK}$ from typical atmospheric variations. Hence, it would be necessary to have a temperature coefficient of less than $0.02 \mathrm{ppm} / \mathrm{K}$ in order to operate a system in this manner without being limited by temperature fluctuations.

From the point of view of reducing the temperature 
coefficient, it is quite possible that a more careful choice of superconducting wire material would yield a smaller value for $\lambda$. Dirty alloys of $\mathrm{Nb}$ should probably be avoided. Also, a detailed study of the magnetic coupling of a finite-size wire winding might lead to optimization of the transformer design to reduce the value of $M_{12 w}$. Thinfilm transformers probably will not offer advantages because the effects of kinetic inductance begin to dominate. However, thick film or tape windings may offer some advantages.

The most straightforward variation in design would be a simple inversion in the roles played by the primary and secondary windings from those here. If the normal metal primary were an inner winding and the secondary superconducting detection winding were outside, there would be no flux density in the superconducting material, and hence no temperature-dependent contribution to $M_{12}$ from $\lambda(T)$. The tradeoff involved in this role reversal would be a probable decrease in the dimensional stability of the critical inner toroid due to the primary being a multilayer winding.

This technique appears well suited for applications as a high-stability dc transfer standard provided the temperature dependence problem can be overcome or significantly ameliorated. Further work in this area will be needed to determine the best approach.

\section{ACKNOWLEDGMENT}

We acknowledge helpful comments and conversations with P. T. Olsen, R. Dziuba, R. Elmquist, A. Clark, R. Steiner, Y. Sakamoto, F. DelaHaye, and K. Weyand.

\section{REFERENCES}

[1] B. F. Field and M. R. McCaleb, "An improved transportable DC voltage standard," IEEE Trans. Instrum. Meas., vol. 38, pp. 324329, Apr. 1989

[2] M. E. Cage, R. F. Dziuba, C. T. VanDegrift, and D. Yu, "Determination of the time-dependence of $\Omega_{\mathrm{NBS}}$ using the quantum Hall resistance," IEEE Trans. Instrum. Meas., vol. 38, pp. 263-269, Apr. 1989 .

[3] D. B. Sullivan, "Superconducting quantum interference devices: An operational guide for rf-biased systems," NBS Tech. Note 629, 1972.

[4] J. C. Gallop, "Use of persistent supercurrents in SQUID current stabilizers and their application to a resistivity measurement on niobium," J. Phys. D, vol. 9, pp. 2111-2115, 1976.

[5] K. Weyand, "DC-current control using SQUID-gradiometer systems," IEEE Trans. Instrum. Meas., vol. IM-29, pp. 324-328, Dec. 1980.

[6] Y. Sakamoto, T. Endo, Y. Murayama, T. Sakuraba, M. Nakanishi. M. Koyanagi, F. Shinoki, H. Nakagawa, and S. Takada, "Josephson array potentiometer at ETL," in Ext. Abst., 1987 Int. Superconductivity Electron. Conf., Tokyo, Japan, Aug. 1987, pp. 84-87.

[7] E. B. Rosa and F. W. Grover, "Formulas and tables for the calculation of mutual and self-inductance," Sci. Papers Bureau of Standards, no. 169, pp. 124-125, 1916.

[8] D. B. Sullivan and R. F. Dziuba, "Low temperature direct curren comparators," Rev. Sci. Instrum., vol. 45, pp. 517-519, Apr. 1974.

[9] K. Grohmann, H.-D. Hahlbohm, and D. Hechtfischer, "The cryo current comparator as a calculable DC ratio standard," IEEE Trans. Instrum. Meas., vol. IM-28, pp. 205-211, Sept. 1979.

[10] R. Meservey and P. M. Tedrow, "Measurements of the kinetic inductance of superconducting linear structures," J. Appl. Phys., vol. 40, pp. 2028-2034, Apr. 1969.

[11] G. K. White, "Thermal expansion at low temperatures of glass ceramics and glasses," Cryogenics, vol. 16, pp. 487-490, Aug. 1976.

[12] A. K. Raychaudhuri, C. Egloff, and L. Rinderer, "Electrodynamics of superconductors. Response to a weak, low-frequency magnetic field," J. Low Temp. Phys., vol. 53, pp. 513-561, 1983.

[13] W. Schwarz and J. Halbritter, "On oxygen enrichments in Nb surface layers," J. Appl. Phys, vol. 48, pp. 487-490, Nov. 1977.

[14] T. P. Orlando, E. J. McNiff, S. Foner, and M. R. Beasley, "Critical fields, Pauli paramagnetic limiting and material parameters of $\mathrm{Nb}_{3} \mathrm{Sn}$ and $\mathrm{V}_{3} \mathrm{Si}$," Phys. Rev. B, vol. 19, pp. 4558-4560, Appendix, 1979. 